\title{
De la selva baja caducifolia al sistema silvopastoril tradicional en la Sierra de Huautla, Morelos
}

\author{
Artemio Cruz León ${ }^{\S}$ \\ Ranulfo Cruz Aguilar \\ ${ }^{1}$ Ciencias en Desarrollo Rural Regional-Universidad Autónoma Chapingo. Carretera México-Texcoco km \\ 38.5, Chapingo, Texcoco, Estado de México. \\ §Autor para correspondencia: acruzl@chapingo.mx.
}

\section{Resumen}

Actualmente La Reserva de la Biosfera de la Sierra de Huautla (Rebiosh) es considerada como el último reducto conservado de la selva baja caducifolia (SBC) de México. En este documento se discute dicho planteamiento y se propone como un agroecosistema tradicional con manejo silvopastoril, con desarrollo histórico y antecedentes de uso previo a la llegada del ganado en la época colonial y una carga animal actual por encima de los parámetros técnicos, además de otros parámetros que fundamentan la creación de un agroecosistema cuyo comportamiento ecológico es cercano a la SBC. Esta situación se complica con la creación de la Rebiosh, cuyo plan de manejo desconoce el uso tradicional de los usufructuarios nativos y se aplican restricciones $y$ criminalización a los usuarios tradicionales; sin considerar formas sustitutas de sufragar las necesidades no cubiertas por las disposiciones del área natural protegida. Se describe el funcionamiento del sistema silvopastoril tradicional y se concluye con la necesidad de profundizar en su comprensión, que permita, mediante procesos participativos, la construcción de aprovechamientos de recursos que fortalezcan sistemas agroforestales tradicionales y la visión de los usuarios a incluir en el Plan de Manejo de la Rebiosh.

Palabras clave: área natural protegida, reserva de la biosfera, sistemas silvopastoriles tradicionales

Recibido: julio de 2020

Aceptado: septiembre de 2020 
La conservación de la naturaleza es uno de los temas de la agenda mundial con alta prioridad internacional, se conciben las áreas naturales protegidas (ANP) como una de las estrategias más utilizadas globalmente para proteger los ecosistemas (Hockings, 2003). Las ANP datan de mediados del siglo XIX y fueron creadas con el objetivo de aislar zonas denominadas 'naturales' pero con la influencia de la sociedad occidental (Durand y Vázquez, 2011).

La justificación en México para la creación de ANP se argumenta por la tasa de deforestación que en 1976 fue de 600000 ha año ${ }^{-1}$, incrementado en 2000 a más de 1000000 ha, de las cuales $60 \%$ son selvas tropicales y subtropical (Velázquez et al., 2002). Para atender estas áreas en alrededor de 30 años el gobierno mexicano conjuntó leyes, políticas e instituciones ambientales (García y Toledo, 2008) y estableció varios tipos de ANP, dando preferencia a la reserva de la biosfera.

Consecuencia de estas acciones, entre 1990 y 2009 el número de ANP paso de 76 a 166, con más de 23000 000, casi el 12\% de la superficie nacional paso a formar parte de alguna de las categorías de ANP (CONANP, 2008). Con esta amplia superficie se pretende resguardar de la biodiversidad, pero ¿Qué pasa con loa usufructuarios de los recursos?, ya que en estas ANP habitan grupos humanos que dependen de los recursos naturales para cubrir sus necesidades básicas, situación que antecede de a la llegada de los españoles.

Con la creación de ANP se incluye a terrenos de propiedad social, que al aplicarlas normas se impacta negativamente las formas de aprovechamiento y conservación tradicional. Desde una perspectiva antropológica las ANP son espacios con una interacción social y científica. Desafortunadamente en realidad se convierten en su contrasentido debido a la imposición por agentes externos a las comunidades y por intereses diferentes de ellas (Descola y Palsson, 2001; Haller y Galvin, 2008) lo resultante es un espacio en conflicto. La implantación de las ANP produce en la actualidad consecuencias negativas para las poblaciones locales (Redford y Fearn, 2007). La prioridad de los intereses biológicos se refleja en que 60\% de las ANP analizadas impidieran el uso del suelo tradicional (Figueroa y Sánchez, 2008), lo cual genera conflictos con los actores locales, por impedimento del uso de recursos en los que se sustenta su sobrevivencia.

Las ANP se rigen mediante un plan de manejo, en la cual se fijan los objetivos que definen los programas, proyectos y las estrategias para su operación. Además, contempla un marco legal basado en la Ley General de Equilibrio Ecológico y Protección al Ambiente (LGEEPA, 2005). Los conflictos con las comunidades son causados por los fundamentos de las políticas públicas: los intereses conservacionistas y la mercantilización de la naturaleza (Hernández y Hernández, 2015).

Desde una postura crítica, en este trabajo se parte de la perspectiva de los actores locales y se establece como hipótesis, la existencia histórica de un sistema silvopastoril en la REBIOSH, mediante el cual se ha realizado un aprovechamiento de los recursos de la vegetación, tierra y agua y por ello actualmente se trata de un agroecosistema modificado producto del manejo de ganado, de la práctica de una agricultura tradicional el aprovechamiento de recursos vegetales y animales, cuya expresión se manifiesta por medio de sistemas que aparentemente guarda similitud con la selva Baja caducifolia.

Así, tenemos una historia regional de uso de los recursos naturales, fauna y flora, que forma parte de la seguridad alimentaria de las comunidades campesinas a las que legalmente pertenece la tierra, existe una tradición de uso racional y visión de conservación, que son características propias de las 
culturas nativas mesoamericanos la cual aún pervive en los campesinos morelenses. La utilización de los recursos naturales se ha ido configurando en los últimos 500 años hacia un aprovechamiento por medio de la ganadería, cuyo resultado se manifiesta mediante la creación de sistemas agrosilvopastoriles tradicionales especializados, cuyos componentes vegetales se asemejan a la selva baja caducifolia y son parte de la cultura rural de la región.

\section{Métodos y técnicas de investigación}

\section{Sitio de estudio}

La Rebiosh se localiza en la parte Sur del estado de Morelos, México, al Sur colinda con los estados de Guerrero y Puebla (Figura 1). Para 2007, 31 comunidades, con 23544 habitantes tenían su territorio o parte de el incluido dentro de las fronteras de la Rebiosh (INEGI, 2006). Los municipios y localidades son: Amacuzac, Casahuatlan y Rancho Nuevo (municipio de Amacuzac); El vergel (municipio de Ayala); Chisco y Vicente Aranda (municipio de Jojutla); La Tigra, El Zapote, Tilzapotla, El Mango, El Salto y Los Tanques (municipio de Puente de Ixtla); Ixtlilco El Chico, Ixtlilco El Grande, El Limón, Los Sauces y Pitzotlan (municipio de Tepalcingo); Ajuchitlan, Santiopa, Chimalactlán, Coaxitlan, Huautla, Huaxtla, Huisaxtla, Nexpa, Xicatlacotla, Pueblo Viejo, Quilamula, Rancho Viejo, San José de Pala y Xochipala (municipio de Tlaquiltenango) (CONANP, 2006).

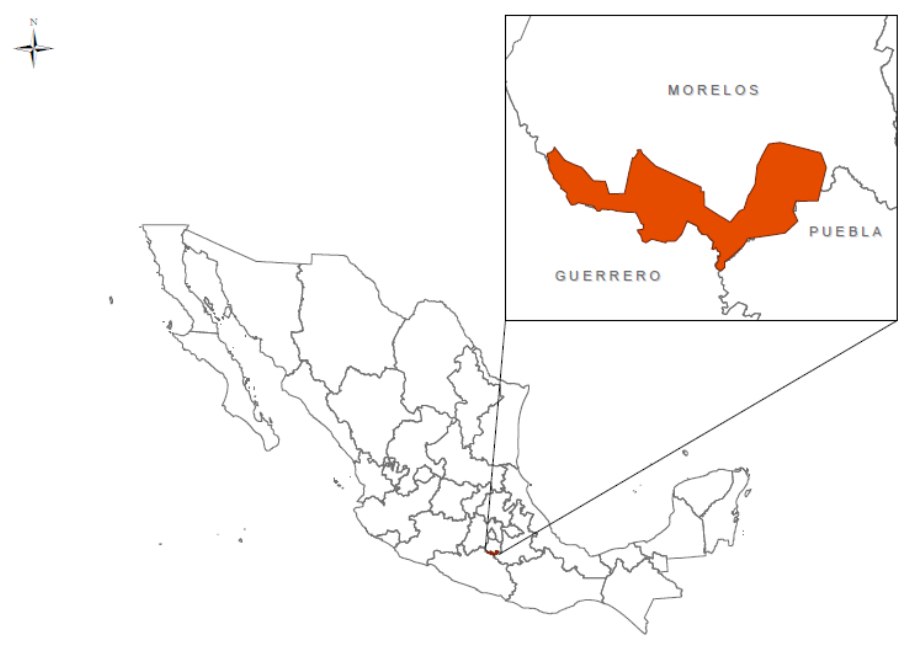

Figura 1. Localización de la Sierra de Huautla. Elaborado con base en CONANP (2006).

\section{Desarrollo metodológico}

Este documento es de tipo descriptivo, inicia con una revisión sobre las ANP como espacios conservados en términos biológicos, y se contrasta con las características de la Sierra de Huautla, como un agroecosistema manejado históricamente como silvopastoril. A partir de este punto central, se desarrollan 4 apartados en torno a la Rebiosh, para explicar las características del funcionamiento del sistema agroforestal tradicional. 


\section{La conservación en las áreas naturales protegidas en México}

Un ANP es una zona dentro del territorio nacional en la cual la nación ejerce su soberanía y jurisdicción, cuyos ambientes originales no han sido significativamente alterados por la actividad del ser humano o que requieren ser preservados y restaurados y están sujetas al régimen previsto (LGEEPA, 2005). En esta definición se hace un énfasis en aislar la biodiversidad del ser humano, por ellos la ANP estará en riesgo y por lo cual es prioridad 'conservar' para preservar y restaurar.

Se plantea dos preguntas ante esta idea ¿Si la tierra fue repartida a los ejidatarios hace casi un siglo, porque ahora la nación debe ejercer su soberanía y legislación sobre un territorio que tiene otro uso? Por otro lado, ¿La Rebiosh cumple la característica de un territorio conservado? Y por último, ¿los grupos humanos que utilizan los recursos son depredadores por definición? Hernández (2015) afirma que en México y a nivel mundial la conservación se ve como una isla separada de la sociedad y por ello hay una profunda desprotección social.

La protección a la naturaleza durante los siglos XVII y XIX fueron encabezadas por la elite social que residía en los países desarrollados, y en vías de desarrollo, aquí es donde se establecen las mayores áreas de conservación, estas acciones también se distinguían por proteger los recursos naturales de los actores locales considerados como una amenaza (Fisher et al., 2008). En el caso de África los cazadores locales eran ubicados como amenaza (Adams y McShane, 1992).

A partir de ello se inició una corriente de ecólogos que, con el uso de tecnología moderna como la fotografía aérea, satelital, sistemas de geoposicionamiento, percepción remota, metodologías cuantitativas y desde una visión vertical (de arriba hacia abajo) identificaban los corredores naturales donde todavía era posible el mantenimiento de la naturaleza, lo que se había mantenido a salvo de la depredación humana (Bennett, 1999). Esta visión sigue dominando, se trata de conservar la vida silvestre a través del diseño de corredores biológicos donde los asentamientos humanos pasan a segundo término. Por lo cual la presencia humana sigue considerándose como perturbadora de las dinámicas ecosistémicas y un peligro para la vida silvestre (Fisher et al., 2008).

En el último tercio del siglo XX a estos espacios se les añadió la característica de belleza escénica (Dudley, 2012), añadiéndole un valor mercantil a los paisajes de las ANP (Hernández, 2007). La conservación en las ANP se adhiere al desarrollo sustentable, mediante la firma del acuerdo denominado Agenda XXI, hecho que sucedió en 1992.

El desarrollo sustentable tiene la finalidad de encontrar una fórmula que sin detener el crecimiento económico logre avanzar en una nueva dirección, en donde las actividades económicas y el manejo (y no sólo aprovechamiento y explotación de la naturaleza) tengan concordancia (Torres, 2013). Su base epistemológica va hacia una ciencia física (economía verde) y no la inclusión de las ciencias sociales (ecología política) (Foldari y Tomassino, 2005). Cabe señalar, que estos modelos de conservación y el modelo de desarrollo están bajo un soporte político para separar la dimensión sociocultural y dejar los recursos naturales a los actores de mayor poder.

\section{La visión biologista en la reserva de la biosfera de la Sierra de Huautla, Morelos}

La Rebiosh como todas las ANP debe regirse mediante un plan de manejo, en la cual se definen los objetivos y se establecen los programas a realizarse que permitan la conservación y en su caso la mercantilización de la naturaleza. En este no están presentes los intereses de los ejidatarios, 
actores locales que, por los siglos, desde sus ancestros han utilizado los recursos y sobrevivido como lo demuestran las zonas arqueológicas y la historia reciente de la región. Este resulta ser el problema fundamental, ya que el funcionamiento de las ANP significa una expropiación de la tierra y recursos, limita el aprovechamiento tradicional y criminaliza el uso que los campesinos hacen, restringen los derechos usufructuarios y es un ataque a la cultura nativa de aprovechamiento de recursos, todo lo cual se traduce en una reducción de la soberanía alimentaria.

De acuerdo con los botánicos del siglo XX la vegetación dominante de REBIOSH es la SBC (Miranda y Hernández, 1963). La superficie original de la SBC fue de $8 \%$ y su extensión abarca desde la costa norte del Pacifico mexicano hasta Panamá (Rzedowski, 1978; Janzen, 1988) y actualmente sólo 2\% de la SBC esta conservado (Janzen, 1988; Murphy y Lugo, 1986). En lo que respecta a la cuenca del Río Balsas la SBC limita la parte norte con los estados de Puebla y Morelos (Rzedowski, 1978).

La Rebiosh, surgió a partir de la iniciativa e interés por parte de algunos académicos de Universidad Autónoma de Morelos (UAEM) donde realizaban estudios taxonómicos (Durand y Vázquez, 2011). Posteriormente la UAEM junto con el Centro de investigación biológica y conservación (CIBYC; antes Centro de Educación Ambiental e Investigación Sierra de Huautla (CEAMISH) y la CONANP, firmaron un convenio de colaboración en 1999 para establecer la Rebiosh (López et al., 2017).

La iniciativa fue de los investigadores y académicos de la UAEM y no de los actores locales, esto confirma lo mencionado por Almazán (2011) donde la conservación es iniciativa de los expertos, que consideran como amenaza las actividades realizadas por los campesinos de la Rebiosh. Por otra parte, los actores locales de la Rebioh tienen estrecha relación con los recursos naturales, a lo largo de la historia han generado simbolismos; sin embargo, la preservación considera como amenaza esos simbolismos.

El plan de manejo de la Rebiosh (CONANP, 2006) tiene en esencia la conservación desde la perspectiva de los investigadores, de esta manera, en los objetivos se establece: preservar los hábitats naturales de la región y los ecosistemas frágiles; conservar la biodiversidad existente; contribuir a la protección de la biodiversidad de la SBC y por último promover el desarrollo económico-social del área, mediante la implementación de proyectos productivos sustentables.

Los objetivos del plan de manejo de la Rebiosh pretenden contrarrestar la siguiente problemática (CONANP, 2006): los procesos de deforestación y cambio de uso del suelo, de zonas forestales a agrícolas y ganaderas; la cacería furtiva y la extracción selectiva de flora y fauna silvestres en estas áreas, así como en las contiguas a las zonas forestadas, al considerar que el aprovechamiento provoca riesgos de perturbación; la pérdida de la práctica agrícola tradicional por una tecnificación y mayor impacto al ecosistema; indefinición en la tenencia de la tierra y falta de aplicación del marco legal ambiental; incidencia de incendios forestales, producto de las prácticas de roza-tumba y quema; falta de vigilancia, falta de instrumentos de planeación adecuados; falta de mecanismos que evalúen la contaminación de cuerpos de agua así como el manejo de residuos sólidos; contaminación de mantos acuíferos y cuerpos de agua, así como algunos efectos en plantas y animales, por actividades mineras. 
En lo que respecta a estudios faunísticos la luciérnaga son las especie más descritas para la Rebiosh, incluyendo los géneros Cratomorphus, Plateros, Photinus y Pyropygodes ( $P$. huautlae), además de un grupo taxonómico de luciérnagas del género Plateros, que se denomina Huautlaensis (Zaragoza, 1999). Existen poblaciones relictuales de 44 especies de mariposas que se han localizado en la vertiente norte de Cerro Frío (Tilzapotla), que son representativas de una etapa geológica cálido-húmeda en esta región y cuya distribución habitual ocurre en la vertiente oceánica de la Sierra Madre del Sur, entre Oaxaca y Nayarit (De La Maza et al., 1995). Por otro lado, registraron 66 especies de mamíferos, 180 especies de aves (50\% de la avifauna conocida de Morelos), 63 especies de reptiles, 11 especies de anfibios y ocho especies de peces (Dorado, 1997).

En lo que respecta a servicios ambientales, la Rebiosh ofrece servicios a los habitantes del sur de Morelos, norte de Guerrero y la Cuenca de Mexcala y Río Balsas. Esto gracias a los escurrimientos superficiales que bajan de las montañas y a su vez abastecen de agua a las presas Emiliano Zapata y Lorenzo Vázquez entre otras (CONANP, 2006). Es posible que la aseveración sobre la biodiversidad argumentada basada en los estudios de la fauna silvestre, y que ha servido para argumentar la creación de la REBIOSH, sea cierta y que dicha fauna habita los sistemas silvopastoriles tradicionales que los productores han generado, los cuales aparentemente reúnen las mismas condiciones ecológicas para estas poblaciones de animales, por ello subsisten y se desarrollan en esta condición modificada de la selva baja caducifolia.

En los estudios sobre la biodiversidad en la Rebiosh se utilizó tecnología para caracterizar a las especies silvestres, gracias a estas herramientas se logró mejor precisión al igual que otras ANP (Bennett, 1999). Pero este diagnóstico no es para el servicio de los actores locales sino para los investigadores y las autoridades gubernamentales, por ello la conservación sigue teniendo la misma esencia de exclusión (Fisher et al., 2008). De acuerdo con los objetivos de la Rebiosh, el plan de manejo contempla varios subprogramas de conservación, en los cuales se hace énfasis en el manejo y turismo, uso público y recreación al aire libre. Estos componentes favorecen los proyectos de ecoturismo que utilizan a la belleza escénica (Dudley, 2012), en donde no necesariamente los beneficiarios son los pobladores locales.

Se han aplicado programas de subsidio y apoyo, entre ellos el Programa de Conservación para el Desarrollo Sostenible (PROCODES), el Programa de Empleo Temporal (PET), el Programa de Conservación de Maíz Criollo (PROMAC) y el Programa de Conservación de Especies en Riesgo (PROCER) (Cruz et al., 2018) que no ha contribuido con abatir la marginación en las comunidades. Además, se han desarrollado talleres sobre el uso de plantas medicinales, el uso de estufas ahorradoras de leña y la construcción de viveros para plantas nativas (Durand y Vázquez, 2011).

En la REBIOSH estas acciones no han impactado lo esperado por los investigadores de la UAEM y las autoridades gubernamentales. Por ello, el presente trabajo sostiene que, ante la falta de eficacia de los programas de gobierno y la academia, habrá que retomar la conservación nativa ancestral, cuyos conocimientos y cosmovisión son una garantía de la conservación, así se ha demostrado en los últimos 9000 años, a pesar de 500 años de coloniaje. El manejo campesino actual es una estrategia de diversificación del uso de recursos y mediante el cual se crea la diversificación de especies domesticadas y silvestres. 


\section{La zonificación del plan de manejo de la reserva de la biosfera de la Sierra de Huautla, Morelos como criterios a discusión}

A partir de la zonificación de la Rebiosh las zonas conservadas corresponden a la clase denominada conservación, que apenas constituye 9.3\% del total del área y a la vez constituye el área de interés de los productores para conservar. El resto es un contrasentido para la conservación, ya que $22.6 \%$ corresponde a zonas perturbadas que las comunidades quieren conservar, 29.2\% corresponden a zonas en donde se permite el pastoreo en forma no intensiva y $36.8 \%$ corresponde a el área en donde se llevan a cabo actividades agropecuarias, esto sumado alcanza $88.6 \%$, cantidad muy elevada si se habla de una ANP.

Por otro lado, la población bovina que pastorea en la Rebiosh es muy elevada. Se considera los datos del último censo agropecuario, en la Rebiosh hay 24668 cabezas de ganado bovino (INEGI, 2007), sin incluir a caballos y mulas ni las otras especies ganaderas y silvestres. Si se considera el índice de agostadero establecido para Morelos, que es de 6.9 ha por unidad animal (SAGARPA, 2014), para mantener esa población bovina se requiere una superficie superior a 170000 ha, lo que significa que la población ganadera bovina es aproximadamente $300 \%$ superior a la carga recomendada. Por ello, no puede considerarse un área conservada y desde luego que las normas de una ANP difícilmente se van a aplicar en una condición como la descrita.

Para entender a la Rebiosh como un sistema agroforestal se puede hacer con un simple recorrido en las áreas en donde pasta el ganado, se aprecia la presencia de pastos, y los árboles dominantes son: cubatas, brasil, huizaches, cuhaulotes, cazahuates, tecolhuixtle y otras especias que se usan como forraje y las especies típicas de la SBC se encuentra en las áreas con mayor pendiente y pedregocidad, a grado tal que el ganado no puede forrajear en esos lugares y por ello se conservan.

\section{La Rebiosh como expresión de un manejo agrosilvopastoril histórico}

Para poner a discusión si la Rebiosh está conservada o no, es importante reconocer que históricamente es un espacio donde los actores locales desarrollaron estrategias de supervivencia desde hace muchos años. Lo cual significa que no se tienen ecosistemas naturales, y en su lugar se han generado agroecosistemas o territorios ocupados históricamente.

Para Hernández y Ramos (1981) el agroecosistema es el ecosistema modificado en menor o mayor grado por el hombre, para utilizar los recursos naturales en los procesos de producción agrícola, pecuaria, forestal o de fauna silvestre. Este concepto se crea asociado al planteamiento de tecnología agrícola tradicional (Hernández et al., 1976; 1977). Actualmente, esta visión histórica puede ser contemplada con los planteamientos de la agricultura comparada desarrolladas por Dumont (1952). A partir de estos enfoques, el espacio ocupado actualmente por la Rebiosh tiene una historia de su uso que incluye miles de años, los ejidatarios actuales manejan el espacio de acuerdo con sus necesidades y con ello modifican la expresión natural de la vegetación, cuyas características actuales difieren de la selva baja caducifolia.

La existencia de grupos humanos en la Rebioh data de hace 22000 años, así lo testifican las pinturas rupestres con esa antigüedad, en los municipios de Puente de Ixtla y Tlaquiltenango (Plancarte y Navarrete, 1911). Los habitantes de estos lugares mantenían contacto con pueblos de la meseta, la huasteca, Guerrero y la mixteca. Estas eran sociedades jerarquizadas y bien organizadas, que 
utilizaban cada palmo de tierra cultivable, se explotaba todo tipo de vegetación y de productos minerales (cal, piedras preciosas, metales nativos de superficie), se cultivaba intensamente el suelo, cazaban y criaban animales de muy diversa índole (CONANP, 2006).

A mediados de 1523, se fundó la Villa de San Francisco Tlaquiltenango, en donde a los españoles se interesaban por los minerales de la plata, en Jojutla el Kaolin y la pizarra negra (Beltrán, 1998). A partir, de esos intereses se desarrollaron las haciendas mineras, algunas de ellas establecidas en 1774, como ejemplos se tienen San Esteban de Pedro Arechardi, Santisima Trinidad de Juan Antonio Pipino, Peñón Grande de José de la Borda, Santiago de Ignacio Loperena, Purísima de Ángel Fernández de Mendoza, San Francisco de Diego Ballesteros y finalmente la Peregrina de Diego Boquedano (Von Mentz, 1977).

La Sierra de Huautla fue ocupada con la ganadería, las grandes haciendas extendieron su territorio por medio de la ganadería bovina extensiva y seguramente équidos, como animales de trabajo usados en labores agrícolas y trasporte. Para resguardo y manejo de la ganadería se fundaron y establecieron caseríos aislados ubicados dentro de la Sierra, que posteriormente dieron origen a poblados actuales, tal es el caso de Huitchila, Los Sauces, El Limón, Ixtlilco el Grande, El Tepehuaje, Sacapalco, Ajuchitlán, San José de Pala. Estos poblados pertenecían a haciendas como Tenextepango, Tenango, Chinameca, entre otras. La ganadería proveía de carne, cueros y de animales de trabajo necesarios en la agricultura, de importancia en la explotación cañera cuya especialidad se concentraba en los valles de Cuautla y Cuernavaca.

\section{EI sistema agrosilvopastoril de la Sierra de Huautla, Morelos}

La ganadería en la Rebiosh determina la dinámica de las comunidades, en torno a ella se organizaba el ciclo agrícola de los cultivos principales, ya que se establece una fecha límite para el momento de siembra, en función de la salida del ganado de los terrenos agrícolas, de la misma manera se hace para la cosecha. Es obligación de los campesinos levantar sus cosechas antes de la introducción del ganado en el otoño-invierno.

Pero también, se relaciona con las actividades festivas y es parte importante en la cultura de las comunidades, la feria del pueblo se hace con toros, son más que eso, la feria son los toros, no se concibe celebración sin esta forma popular de diversión, que ha evolucionado de usar animales locales con una serie de rituales y organización de la comunidad, al uso de animales de 'juego', animales especializados por selección específica, montados ya no por los jóvenes más valientes de la localidad, sino por montadores profesionales, cuya presentación es todo un espectáculo, con altos costos, mismos que paga el pueblo y la organización local de la feria.

La ganadería se practica en toda la zona de estudio, es una ganadería rotativa en función de la disponibilidad de forraje y agua para abrevaderos, y por lo tanto de la época del año. En la época de lluvias (de junio a noviembre) se ubica en la zona cerril, en lo que son los usos comunes de los ejidos, durante la época de secas (diciembre a marzo) baja a los terrenos de cultivo de temporal, a consumir los esquilmos agrícolas de sorgo o maíz y los meses restantes (abril a mayo), puede estar en los terrenos agrícolas o en los corrales del productor en donde recibe forraje o suplementos (Figura 2). 


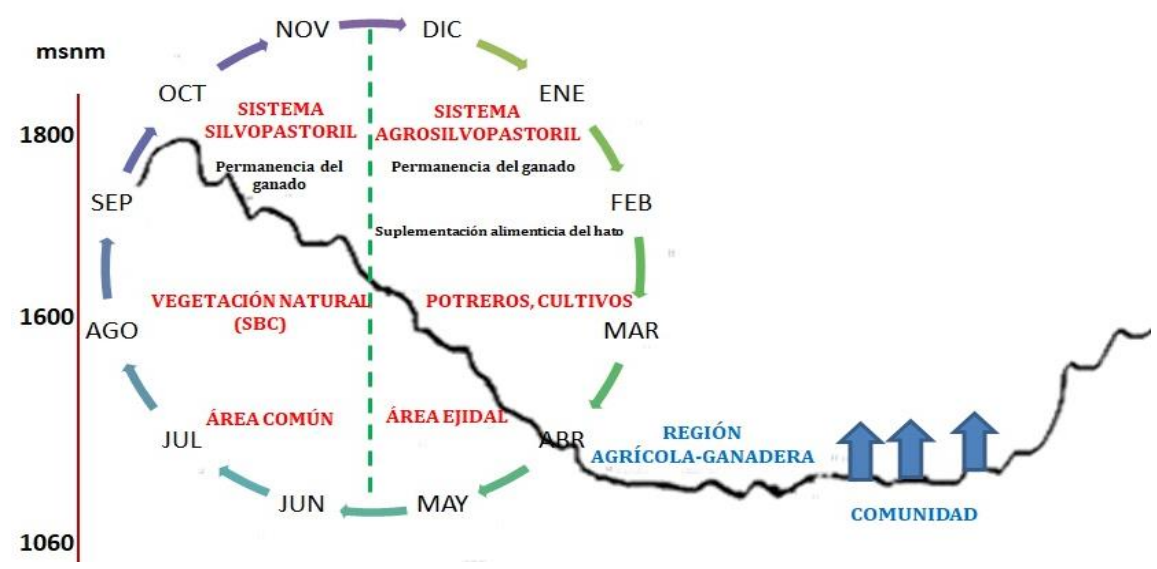

Figura 2. Manejo rotacional y permanencia del ganado a través del año en las diferentes áreas del ejido de las comunidades de la Sierra de Huautla (Burgos et al., 2016; Uribe et al., 2015).

Las condiciones ambientales de las comunidades de la Sierra de Huautla son contrastantes entre sí. Con la finalidad de explicar las diferencias y trasmitir sus implicaciones para los ejidatarios y sus significados en la producción agrícola y ganadera, se intentan resumir en tres diferentes. La primera corresponde a las comunidades cuya ubicación se encuentra al interior de la Sierra, y por ello, sus recursos naturales, áreas para desarrollar las actividades productivas contrastan con el resto de las comunidades. Se aprecia la ausencia de terrenos planos, los lomeríos menos pronunciados se utilizan para la agricultura con roturación, los terrenos con cubierta vegetal se emplean como agostadero. Las comunidades con este tipo de explotación son: El Limón, Los Sauces, El tepehuaje, San José de Pala, Ajuchitlán, Santiopa, Huautla, Xochipala, Rancho Viejo, entre otros.

Las comunidades que poseen un territorio que combina terrenos con pendiente elevada, lomerío y áreas planas tendrá condiciones de producción menos limitantes, ya que sus terrenos planos permitirán uso de riego y combinar los avances tecnológicos como el riego, semillas mejoradas y plaguicidas sin restricción. Para el caso de terrenos con mayor pendiente se realiza una agricultura de temporal con restricciones por pendientes elevadas, suelos someros, resultante en una agricultura marginal, de autoconsumo o con parte de la cosecha para la venta.

En el caso de terrenos con mayor pendiente, sus características obligan a un uso silvopastoril, aprovechamiento de la vegetación y minerales. Las comunidades con este tipo de condición son: Ixtlilco el Grande, Ixtlilco el Chico, Tepalcingo, Pitzotlán, Huitchila, Zacapalco, Chinameca, El Vergel, Santa Cruz, entre otros. Por último, la tercera condición corresponde a comunidades, que no tienen terrenos dentro de la Sierra de Huautla; sin embargo, por su cercanía y dado que no poseen terrenos cerriles, útiles para el pastoreo del ganado en la época de lluvias, hacen arreglos con comunidades de la Sierra y establecen convenios de aparcería para que su ganado permanezca en los terrenos de agostadero durante el periodo de lluvias, a cambio de algún pago. Las comunidades con este tipo de convenio son: Atotonilco, Huitchililla, Tetelilla, Villa de Ayala.

Los campesinos en general tienen una comprensión bastante desarrollada y conocen características físicas que tienen las especies arbóreas, atribuidas a evidencias empíricas, supuestos y creencias (Muñoz et al., 2003). El ganado consume más las especies arbóreas que las herbáceas, a ello se 
debe un amplio conocimiento sobre la fisiología, adaptación y otros aspectos ecológicos y socioeconómicos sobre las diversas especies arbóreas para la generación de bienes y servicios, lo cual permite a los productores un uso apropiado en la cotidianidad agroforestal (Mosquera, 2010).

Clasificar y caracterizar a las especies desde un enfoque biologista es limitado para integrar las perspectivas de los campesinos y esta situación llevó a un distanciamiento entre actores locales y externos, por lo cual es importante aplicar los métodos cualitativos y cuantitativos de investigación (Pimbert, 1995).

Finalmente, en el ejido de Ajuchitlan, Tlalquitenango, Morelos, Burgos et al. (2016) define dos tipos de valor cultural (IC) y valor de importancia (VI), en lo que respecta a las especies más importantes por su IC, estas fueron Eysenhardtia polystachia con 90\%, Mimosa benthamii con $82 \%$, Haematoxilum brasiletto con $68 \%$ y Crescentia alata con $57 \%$, en tanto que las especies con mayor VI fueron: Mimosa benthamii con $114 \%$ y Crescentia alata con 50\%, entre otras.

Estas especies tiene un aprovechamiento de resina, medicinal, postes, frutos, forraje, leña y cerco vivo, y conocerlas puede ayudar a la construcción de proyectos de sistemas agrosilvopastoriles con los campesinos (Briñez et al., 2016). La importancia de estas especies muestra su dominancia en el sistema silvopastoril, y afirma la diferencia de este agroecosistema con la dominancia de especies vegetales propias de la SBC.

\section{Conclusiones}

La expresión actual de la vegetación de la Rebiosh es la de un sistema silvopastoril, diferente a la expresión de la SBC, mismo que se ha formado históricamente en casi cinco siglos siendo el ganado bovino una constante en la explotación de los recursos naturales que ha pasado de la ganadería bovina extensiva, heredada por los haciendas españolas, trasferida a los grandes hatos en las haciendas porfirista, que fueron destruidas por la revolución Zapatista y a la vez fueron sus sustento, y que resurge en la ganadería ejidal.

La vegetación de la Rebiosh no es un ecosistema conservado, que fue una de las justificaciones para la creación del área natural protegida, con lo cual el neoliberalismo abre la puerta a la mercantilización de la naturaleza y de la biodiversidad, estrategia en la cual los dueños del territorio (ejidatarios) no fueron consultados y tan poco son los beneficiarios. Los aprovechamientos tradicionales de recursos naturales son criminalizados, se condicionan a la aplicación de la norma, y con ello se reduce las decisiones comunitarias de manejo a la burocracia institucional, que no se pone al servicio de los usuarios y reduce sus decisiones a la aplicación de una ley.

La elevada carga animal que soporta la SH puede explicarse en términos de la utilización de espacios con esquilmos agrícolas y la suplementación temporal en la época limitante de producción de forraje, de manera cíclica a lo largo del año. Expresiones todas ellas del manejo campesino de la ganadería que ha logrado utilizar eficientemente los recursos naturales y se expresan a través de sistemas silvopastoriles tradicionales. Por esta razón, los sistemas silvopastoriles tradicionales deben de estudiarse a detalle y junto con los productores construir propuestas de mejoramiento de ellos y su inclusión como alternativa al Plan de Manejo de la Rebiosh, en donde la perspectiva de los usuarios se incorpore. 


\section{Literatura citada}

Adams, J. S. and McShane, T. O. 1992. The myth of wild Africa: conservation without illusion. California, EUA, Univ. of California Press. 217 p.

Beltrán, G. R. 1998. Generalidades sobre la historia de Tlaquiltenango, Tlayehualco y Xoxocotla. México, Dirección General de Culturas Populares, PACMYC. 55 p.

Bennett, A. F. 1999. Enlazando el paisaje. El papel de los corredores y la conectividad en la conservación de la vida Silvestre. Clayton, Australia, Facultad de Ecología y Medio Ambiente. 277 p.

Briñez, A. S.; Rodríguez-Paola, R. P. y Mora, D. J. 2016. Conocimiento local de especies leñosas y herbáceas usadas en alimentación de ovinos en el norte del Tolima. Agroforestería Neotropical. 1(6):25-33.

Burgos, H. B.; Cruz, L. A.; Uribe, G. M. L. B. A. y Torres, R. M. 2016. Valor cultural de especies arbóreas en sistemas agroforestales de la Sierra de Huautla, Morelos. Rev. Mex. Cienc. Agríc. 16(especial):3277-3286.

Coad, L.; Campbel, A.; Miles, L. and Humphries, K. 2008. The costs and benefits of protected areas for local livelihoods: a review of the current literature. Cambridge, RU. UNEP World Conservation Monitoring Centre. $32 \mathrm{p}$.

CONANP. 2006. Programa de Conservación y Manejo de la Reserva de la Biósfera Sierra de Huautla. México, Comisión Nacional de Áreas Naturales Protegidas. 204 p.

CONANP. 2008. Comisión Natural de Áreas Naturales Protegidas ¿Qué son las AP? México, Comisión Nacional de Áreas Naturales Protegidas. http://www.conanp.gob.mx/q-anp.html.

Cruz, A. R.; Cuevas, R. V.; Cruz, L. A. y Ramírez, V. B. 2018. Impacto social de la mercantilización de la naturaleza en la Sierra de Huautla, Morelos. Estudios sociales. 28(51):2-23. http://dx.doi.org/10.24836/es.v28i51.521.

De La Maza, E. R. A.; White, L. y De La Maza, R. R. 1995. Exploración de factores compensatorios que permiten el refugio de rhopalocerofauna higrófila en cinco cañadas de clima subhúmedo en Morelos, México. Rev. Soc. Mex. Lep. 16(1):1-63.

Descola, P. y Palsson, G. 2001. Introducción. In: Descola, P. y Palsson, G. (Eds.). Sociedad y naturaleza. Perspectivas antropológicas. Siglo XXI. México, DF. 11-36 p.

Dorado, R. O. R. 1997. Inventario florístico de la Sierra de Huautla, Morelos. Centro de Educación Ambiental e Investigación Sierra de Huautla. Universidad Autónoma del Estado de Morelos. Informe final SNIB-CONABIO proyecto. México, DF. http://www.conabio.gob.mx/institucion/proyectos/resultados/InfB054.pdf.

Dudley, N. 2012. Introduction: understanding the biodiversity values of category V protected areas. In: Dudley, N. and Sue, S. (Eds.). Protected landscapes and wild biodiversity. United Kingdom, IUCN. 7-18 p.

Dumont, R. 1952. Agricultue comparée. Larousse Agricole. 903-938 pp.

Durand, S. L. y Vásquez, H. L. B. 2011. Discursos sobre participación social en la Reserva de la Biosfera Sierra de Huautla, Morelos. In: Argueta, V. A.; Corona, M. E. y Hersch, P. (Coords.). Saberes colectivos y diálogo de saberes en México. México, CRIM-UNAM. 101-120 p.

Figueroa, F. and Sánchez, C. V. 2008. Effectiveness of natural protected areas to prevent land use and land cover change in Mexico. Biodiv. Cons. 17(13):3223-3240. 
Fisher, R. J.; Maginnis, W. J. S.; Barrow, J. E. y Jeanrenaud, S. 2008. Pobreza y conservación: Paisajes, pueblos y poder. San José, Costa Rica, Unión Mundial para la Naturaleza, IUCN. $168 \mathrm{p}$.

Foldari, G. y Tommasino, G. 2005. El enfoque técnico y el enfoque social de la sustentabilidad. México, Miguel Ángel Porrúa-Universidad Autónoma de Zacatecas. 197-206 p.

García, F. E. y Toledo, V. M. 2008. Evaluación de sistemas socioecológicos en áreas protegidas: un instrumento desde la economía ecológica. Argumentos, 21(56):103-116.

Haller, T. and Galvin, M. 2008. Participation, ideologies and strategies: a comparative new institutionalist analysis of community conservation. In: Galvin, M. and Haller, T. (Eds.). People, protected areas and global change. Berna, Suiza, University of Bern. 507-551 pp.

Hernández, L. J. J. 2015. El paisaje agavero: expansión y estatización. Ecología cultural política y nuevas formas de creación de valor. Tesis de doctorado. Zamora, Michoacán. El Colegio de Michoacán. 225 p.

Hernández, X. E. y Ramos, R. A. 1981. Metodología para el estudio de agroecosistemas con persistencia de tecnología agrícola tradicional. In: Hernández, E. X. (Ed.). Agroecosistemas de México, contribuciones a la enseñanza, investigación y divulgación agrícolas. Chapingo, México, Colegio de Postgraduados. 321-333 pp.

Hernández, X. E.; Inzunza, F. M.; Solano, B. S. y Brauer, F. G. 1976. Estudio de la tecnología agrícola tradicional. Avances en la enseñanza y la investigación 1976-1977. México, Colegio de Postgraduados. 27-30 pp.

Hockings, M. 2003. Evaluating the management of protected areas: integrating planning and evaluation. Environ. Manag. 22(3):337-345.

INEGI. 2006. Instituto Nacional de Estadística Geografía e Información. XII Censo General de Población y Vivienda. Aguascalientes, México. Instituto Nacional de Estadística, Geografía e Informática. https://www.inegi.org.mx/programas/ccpv/2000/.

INEGI. 2007. Censo Nacional Agropecuario 2007. México, Laboratorio de microdatos, INEGI.

Janzen, D. 1988. Tropical dry forests, the most endangered major tropical ecosystem. In: Wilson, E.O. and Peter, F. M. (Eds.). Biodiversity. Washington, D.C: National Academy Press. 130-137 p.

Ley General de Equilibrio Ecológico y Protección al Ambiente. 2005. Ley General de Equilibrio Ecológico y Protección al Ambiente. Última reforma DOF 23-02-2005. Cámara de Diputados. México, DF. http://www.diputados.gob.mx/LeyesBiblio/pdf/148_050618.pdf.

López, M. X.; Vázquez, L. B.; Valenzuela, G. D.; Wehncke, E.; Maldonado, A. B. y Durand, S. L. 2017. Percepciones de los habitantes de la reserva de la biósfera Sierra de Huautla: hacia el desarrollo de nuevas estrategias de manejo participativo. Interciencia. 42(1):8-16.

Miranda, F. y Hernández, X. E. 1963. Los tipos de vegetación de México y su clasificación. Bol. Soc. Bot. Méx. 28:291-279.

Mosquera, D. H. 2010. Conocimiento local sobre bienes y servicios de especies arbóreas y arbustivas en sistemas de producción ganadera de Rivas, Nicaragua. Tesis de Maestría. Turrialba, Costa Rica, CATIE. 91 p.

Muñoz, D.; Harvey, C. A.; Sinclair, F. L.; Mora, J. e Ibrahim, M. 2003. Conocimiento local de la cobertura arbórea en sistemas de producción ganadera en dos localidades de Costa Rica. Agroforestería en las Américas. 10(39-40):61-68.

Murphy, P. G. and Lugo, A. E. 1986. Ecology of tropical dry forest. Annual review of ecology and systematics. 17(1):67-88.

Pimbert, M. 1995. The Need for another research paradigm. Seedling. 11(2):20-26. 
Plancarte y Navarrete, F. 1911. Tamoanchan, el estado de Morelos y el principio de la civilización. Mexico, El Mensajero. 194 p.

Redford, K. H. and Fearn, E. 2007. Protected areas and human displacement: a conservation perspective. USA, Bronx, New York, Wildlife Conservation Society. 152 p. http://indiaenvironmentportal.org.in/files/Protected\%20Areas\%20and\%20human\%20disp lacement.pdf.

Rzedowski, J. 1978. Vegetación de México. México, DF. Limusa. 432 p.

SAGARPA. 2014. Secretaría de Agricultura, Ganadería, Desarrollo Rural, Pesca y Alimentación Comisión Técnico Consultiva de Coeficientes de Agostadero (COTECOCA). http://www.sagarpa.gob.mx/desarrolloRural/Publicaciones/Lists/CursoTaller\%20Desarrol lo\%20de\%20capacidades\%20orientadas\%20a/Attachments/35/10.pdf.

Torres, C. G. 2013. El desarrollo sustentable en México (visión crítica hacia un desarrollo compatible). Universidad Autónoma Chapingo. Texcoco, Estado de México. 259 p.

Uribe, G. M.; Cruz, L. A.; Juárez, R. D.; Lara, B. A.; Romo, L. J. L.; Valdivia, A. R. and Portillo, V. M. 2015. Diagnosis importance of rural development of a model agroforestal in peasant communities of Sierra de Huautla. Revista Ra Ximhai. 11(5):189-200.

Velázquez, A.; Mas, J. F.; Mayorga, S. R.; Díaz, J. R.; Alcántara, C.; Castro, R.; Fernández, T.; Palacio, J. L.; Bocco, G.; Gómez, R. G.; Luna G. L.; Trejo I.; López, G. J.; Palma, M., Peralta, A.; Prado, M. J. y González, M. F. 2002. Estado actual y dinámica de los recursos forestales de México. Biodiversitas. 6(41):8-15.

Von-Mentz, B. 1977. Haciendas de Morelos. México, Instituto de Cultura, Consejo Nacional para la Cultura y las Artes, Editorial Porrúa. 412 p.

Zaragoza, C. S. 1999. Cantharoidea (Coleoptera) de México. III. El género Plateros Bourgeois (Lycidae: Erotinae: Platerodini). Acta Zool. Mex. 78:1-71. 